\title{
Periodontally involved teeth - extract or not?
}

\section{Abstracted from \\ Popelut A, Rousval B, Fromentin O, Feghali M, Mora F, Bouchard P.}

Tooth extraction decision model in periodontitis patients. Clin Oral Implants Res 2010; 21: 80-89.

Address for correspondence: Philippe Bouchard, Department of Periodontology,

Service of Odontology, Hôtel-Dieu Hospital, AP-HP, Paris 7-Denis Diderot University,

U.F.R. of Odontology, Paris, France. E-mail: phbouch@noos.fr

\section{Question: Can a decision tree assist in deciding whether to extract a periodontally involved tooth?}

Design Decision analysis modelling.

Method The authors created a scenario in which a patient with a periodontally involved tooth was referred for a decision to be made over whether to extract the tooth or not. Neighbouring teeth were healthy. A decision tree that included no treatment, periodontal treatment, extraction and replacement with fixed partial denture, and extraction with single implant was created. It was populated with 'objective' probability data from systematic reviews and 'subjective' probability data from clinicians. No patient preference data were included. Eligible studies had to have a follow-up of $\geq 5$ years. Four 'senior experts' including three periodontists and one prosthodontist evaluated a quantitative measure of the strength of the preference for an outcome.

Outcome measure Expected utility (EU) of each management approach on a scale of 0-100.

Results The EU could not be calculated for the no treatment option owing to lack of probability data (ie no-one has followed up patients who do not receive periodontal treatment). For the periodontal treatment, FPD and implant groups the respective EU intervals over five years were 79-96, 86-89 and 94-95.

Conclusions The EU intervals suggest the implant option is better than the FPD option if the tooth is extracted, but it is unclear whether periodontal treatment or extraction and implant is best.

\section{Commentary}

Decision Tree Analysis is a probabilistic approach for optimising decision-making in a world of uncertainty and choice. In the absence of well designed randomised controlled trials, this approach has been shown to offer a valid alternative for guiding clinical decision making. ${ }^{1,2}$ The validity of a decision tree analysis is judged on how well the model reflects the real life trade-offs between the positive outcomes (ie, benefits) and negative outcomes (ie, risks) of each clinical option.

Popelut et al. used this approach to determine the best way to manage a periodontally compromised tooth. ${ }^{3}$ They compared the outcomes of four alternative therapy options for this clinical scenario: (1) no treatment, (2) periodontal treatment, (3) tooth extraction and replacement with a conventional dental bridge or (4) an implant supported crown. The authors concluded that the implant supported crown was the treatment of choice if the tooth must be extracted.

A significant limitation of Popelut et al.'s decision model is that it only considers the positive outcomes and none of the risks associated with the proposed treatment options. For example, dental implantation is a surgical technique that places the patients at risk of mandibular nerve damage or maxillary sinus complication. ${ }^{4,5}$ Sensory damage to the lower lip more than one year after implant insertion has been reported to be between 1-7\%. ${ }^{6}$ Although the risks may be low, the impact - and thus the patient perceived utility - is significant and likely to be a factor in any decision to undergo such procedures.

Also, the analysis of the baseline data is susceptible to bias. For example, the authors presented no pooled estimates and confidence intervals for any option; rather they presented the data as a range. They found that the lower limit 5-year survival rate for the implant option (96.8\%) was higher than the upper limit 5-year survival rate for the dental bridge option (96.4\%), inferring that the implant option has the favourable prognosis. However, they excluded one study from their systematic review, one by Salinas \& Eckert (2007) that reported comparatively similar pooled estimate success rates for the conventional dental bridge (94.0\% [CI $91.6-96.4 \%]$ ) and the implant supported crown (95.1\% [CI-92.2\% $-98.0 \%])^{7}$

Furthermore, the authors used utilities taken from a consensus of four dental specialists (three of whom were periodontists), even though patient centred utilities for conventional bridges and implant supported crowns are available in the literature. ${ }^{8}$ Patient centred utilities have been shown to be significantly different from 
those reported by dentists. ${ }^{9}$ As such, the utility data of each outcome used in this analysis are at risk of specialty bias in favour of treatment performed by periodontists.

Finally, this analysis does not present an acceptable sensitivity analysis that accurately reflects the reliability of the decision tree results in a world of imperfect information. The authors refer to a 'robustness calculation' for the 5-year survival rate for the 'no treatment' option as being $78 \%$. This value does not reflect on the robustness, or sensitivity, of the result of the decision model, but rather the threshold above which the 'no treatment' option is favoured. A sensitivity analysis is typically presented graphically for the reader to assess how well (ie, robustness), or not (ie, sensitivity), the result of the decision analysis holds up to varying the values of one or more of the variables. ${ }^{10}$ The authors' conclusion favours the implant supported crown, yet no sensitivity analysis is given on how this option holds up to other alternatives when the survival rate or utility vary from baseline values .

It appears that Popelut et al.'s proposed decision analysis is biased in favour of the implant option at multiple stages. As such, this analysis does not offer a valid decision analysis to guide clinicians or policy makers on the management of a periodontally compromised tooth.
Ben Balevi

Faculty of Medicine, University of British Columbia, Vancouver, British Columbia, Canada.

1. Bress JN, Hulgan $T$, Lyon JA, Johnston CP, Lehmann H, Sterling TR. Agreement of decision analyses and subsequent clinical studies in infectious diseases. $\mathrm{Am}$ Med 2007; 120: 461

2. Claxton K, Ginnelly L, Sculpher M, Philips Z, Palmer S. A pilot study on the use of decision theory and value of information analysis as part of the NHS Health Technology Assessment programme. Health Technol Assess 2004; 8: 1-103.

3. Popelut A, Rousval B, Fromentin O, Feghali M, Mora F, Bouchard P. Tooth extraction decision model in periodontitis patients. Clin Oral Implants Res 2010; 21: 80-89.

4. Palma-Carrió C, Balaguer-Martínez J, Peñarrocha-Oltra D, Peñarrocha-Diago MA. Irritative and sensory disturbances in oral implantology. Literature review. Med Oral Patol Oral Cir Bucal 2011; 16: e1043-1046.

5. Galindo-Moreno P, Padial-Molina M, Avila G, Rios HF, Hernández-Cortés P, Wang HL. Complications associated with implant migration into the maxillary sinus cavity. Clin Oral Implants Res 2011; 15. doi: 10.1111/j.1600-0501.2011.02278.x.

6. Greenstein G, Tarnow D. The mental foramen and nerve: clinical and anatomical factors related to dental implant placement: a literature review. J Periodontol 2006; 77: 1933-1943

7. Salinas TJ, Eckert SE. In patients requiring single-tooth replacement, what are the outcomes of implant- as compared to tooth-supported restorations? Int J Oral Maxillofac Implants 2007; 22: 71-95.

8. Balevi B, Shepperd S. The management of an endodontically abscessed tooth: patient health state utility, decision-tree and economic analysis. BMC Oral Health 2007; 7: 17.

9. Fyffe HE, Kay EJ. Assessment of dental health state utilities. Community Dent Oral Epidemiol 1992; 20: 269-273.

10. Krahn MD, Naglie G, Naimark D, Redelmeier DA, Detsky AS. Primer on medical decision analysis: Part 4--Analyzing the model and interpreting the results. Med Decis Making 1997; 17:142-151.

Evidence-Based Dentistry (2011) 12, 110-111. doi:10.1038/sj.ebd.6400826 